\title{
Development of a Dummy Bone Graft Material from Egg-Shells for Practice on Cadaver Animal Model
}

\author{
T K Pal ${ }^{1 *}$, Suchibrata Bhar ${ }^{2}$ and Goutam Das ${ }^{3}$ \\ ${ }^{1}$ Department of Periodontics, Guru Nanak Institute of Dental Sciences \& Research, India \\ ${ }^{2}$ Postgraduate Trainee, India \\ ${ }^{3}$ Research Associate, India
}

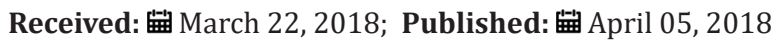

*Corresponding author: T K Pal, Department of Periodontics, Guru Nanak Institute of Dental Sciences \& Research, India

\begin{abstract}
Aim: Additive osseous surgery requires graft material to be placed in osseous defects or for sinus lifting procedure. Placement of graft material in dummy jaw or on cadaver jaw by students/learners for practice is needed before actual placement on patients. There is a need to develop a dummy radio-opaque material for use as a practice material by students or learners in defects of dummy jaw or on cadaver jaw.
\end{abstract}

Materials and methods: Dummy graft material was prepared from discarded egg-shells (waste) and was used for sinus lifting in a cadaver goat jaw as taste case.

Result: A measureable radio-opacity of egg-shell particles was seen below the sinus floor after sinus lifting on a cadaver goat jaw.

Conclusion: Radio-opaque egg-shell graft material can be used as dummy graft material for practice as a teaching material.

Keywords: Osseous defects; Egg-Shell; Cadaver goat; Dummy Graft material

\section{Introduction}

The development of biomaterials for bone tissue replacements in dentistry has attracted a lot of interest among professionals due to the rise in the number of patients requiring bone replacements 1. The bony craters and other defects in the bony architecture like insufficient ridge height for proper placement of implants may be managed by suitable substitutes to make up for the lost structures. During teaching and training programme, each student or trainee should be given a cadaver model on which a dummy bone graft materials should be used for practice before actually performing surgery on patients. Egg-shells has been thought of as the material of choice in terms of the fact that it is made up of calcium carbonate and easily procurable. The particles from the egg-shells could serve the purpose of a dummy graft material which can be used by learners on animal cadaver models in hands-on-training [1,2].

\section{Aims}

Bony defects around tooth and or implant needs additive osseous surgery to improve prognosis of tooth or implant. Additive osseous surgery requires graft material to be placed in osseous defects or for sinus lifting procedure. Placement of graft material in dummy jaw or on cadaver jaw by students/learners for practice is needed before actual placement on patients' mouth. There is a need to develop a dummy radio-opaque material for use as a practice material by students or learners in defects of dummy jaw or on cadaver jaw.

\section{Material and Methods}

\section{Preparation of dummy graft material}

The material used for this study was obtained from the discarded waste shells of poultry eggs (Figure 1). After proper washing with clean running tap water with soap, the shells were freed from the adherent protein coat and then, dried in bright sunlight before pulverizing into fine powder with the help of kitchen grinder (Figure 2). It was passed through a sieve used in kitchen for obtaining desired dimension of particles (Figure 3). The dummy graft was stored in clean, dry container to avoid moisture. 
The appearance of the powder after preparation was white and granular in texture of approximately 100 to300 mesh [3].

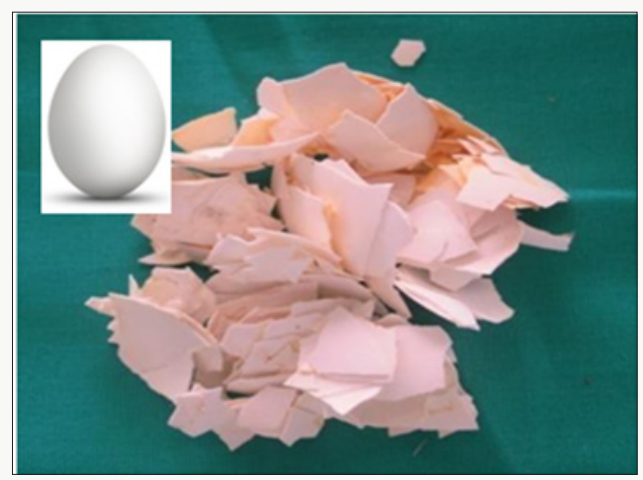

Figure 1: Egg-shells, (Egg in inset).
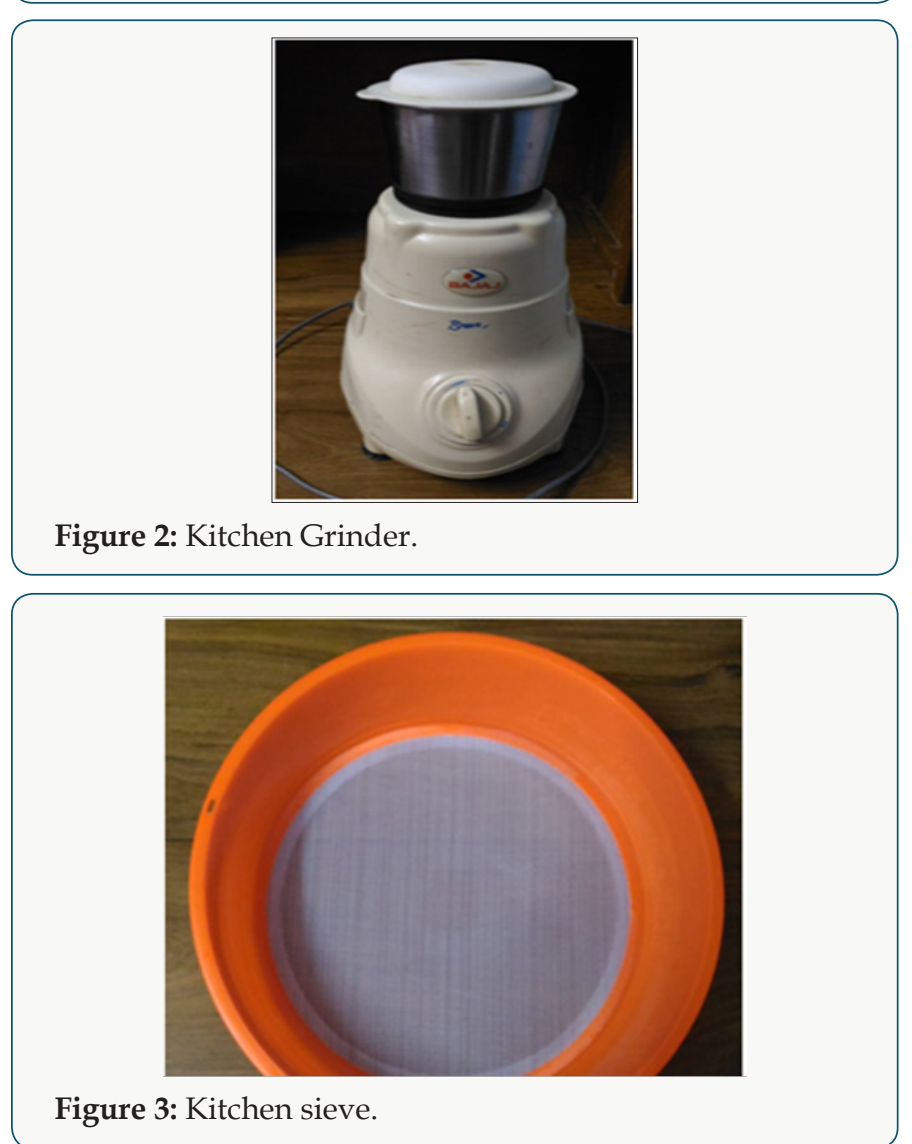

\section{Composition}

It is made up of $94 \%$ CaCo32.

\section{Physical characteristics}

The handling properties of this newly developed dummy egg shell graft exhibited the same characteristics (like consistency and free-flowing nature) as the actual ones available in the market, especially which are made up of calcium compounds.

\section{Radiographic characteristics}

Comparison of radio-opacity with other Ca- based materials like chalk dust, TCP, and egg shell were done. Each of these products was poured into empty disposable plastic needle covers (Figure 4). The plastic covers were then attached to IOPA film with help of adhesive transparent tape and properly marked on each plastic cover. This was then exposed to X-Ray radiation. The radiograph was analyzed visually for relative radio-opacity of each material [4] (Figure 5).
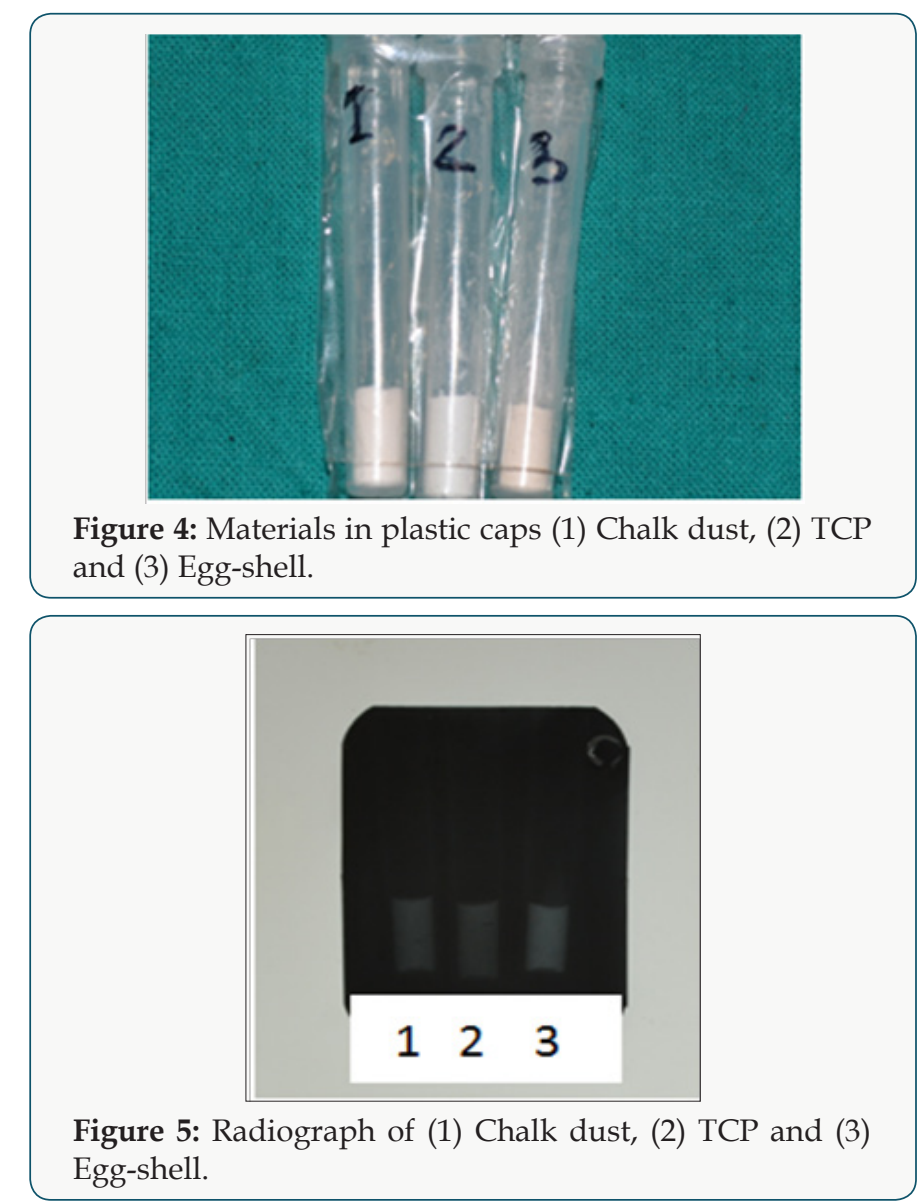

\section{Test on Goat cadaver for ridge enhancement by sinus lifting}

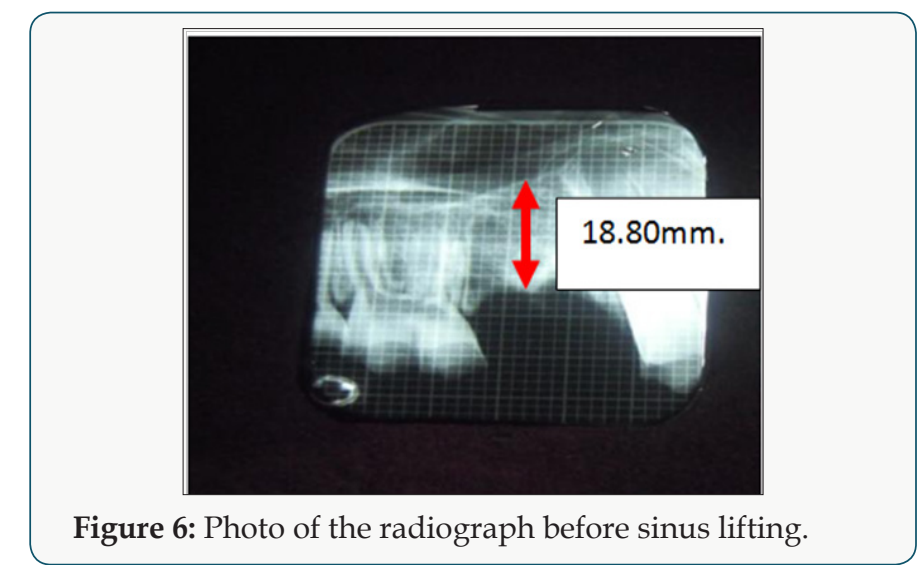

Cadaver Goat head was procured from slaughter house and a sagital split was made. Radiograph was taken before attempting ridge enhancement as base line with the help of a grid. The distance from the crest of ridge to the floor of maxillary sinus was measured 
(Figure 6). Sinus lifting was done appropriately using appropriate tools and graft material was placed cautiously into the space below the sinus membrane. Again, radiograph was taken finally with the help of a grid and the distance from the crest of the ridge to the new floor of the sinus measured [5] (Figure 7).

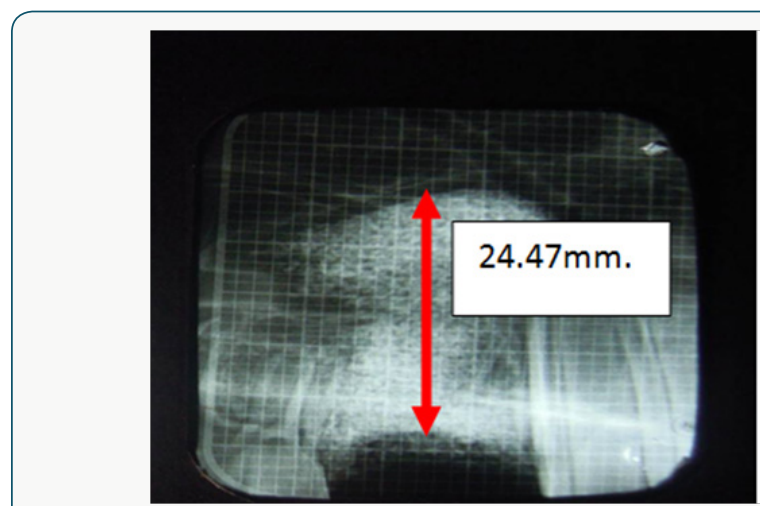

Figure 7: Photo of the radiograph after sinus lifting.

\section{Results and observation}

After the radiograph was taken, it was clearly found that the radio-opacities of the three materials taken under consideration were distinctly different from each other, and the eggshell powder was found to be more radio-opaque than the other materials. The distance for the alveolar crest to the floor of the sinus and distance from the alveolar crest to the elevated floor of the sinus were 18.80 $\mathrm{mm}$. and $24.47 \mathrm{~mm}$. respectively (Figures 6 \& 7). The net gain in bone height was $5.67 \mathrm{~mm}$.

\section{Discussion}

Poultry-egg consumed as food for protein is encased in a hard shell which is a waste product after use and is thrown away as a biologic waste. This waste material is thought of to be used as a dummy graft in the present study model for filling of bone defects and for sinus augmentation. The shell contains about $94 \%$ of calcium carbonate (CaCO3) by weight as the active ingredient 2.This can be seen radiographically as a radio-opaque substance and thus acceptable to be seen post-operatively in radiographs, an essential characteristic of a graft material. The students intended to learn the handling of graft material during placement of the material into osseous defects or for sinus lifting procedures may require a preclinical practice into defects of dummy jaws or in cadaver jaws. This may facilitate them to learn how better the graft material can be placed or utilized otherwise. This will not only enhance their confidence but also solve some of the teachers' concern about catering to the practical pre-clinical demonstration .The procurement of egg-shells and preparation of graft particles is beyond the need of seeking permission from any ethical committees and does not encroach upon any ethical issues.3-6 Use of such an easily available waste material as dummy graft material for practising on dummy jaw or cadaver animal models would be helpful for the beginners [6].

\section{Conclusion}

From the study it is clear that egg-shell waste material can be used as dummy graft material for performing various grafting procedures, including sinus lifting in hands-on practice on dummy jaw or on cadaver models.

\section{References}

1. Pearce AL, Richards RG, Milz S, Schneider E, Pearce SG (2007) Animal model for implant biomaterial research in bone. A review: European cells and Materials 13: 1-10.

2. Lechtanski VL (2000) Calcium carbonate content of Egg-shells. InquiryBased Experiments in Chemistry, Oxford: New York, USA 159-165.

3. Pal S, Pal TK (1997) In: Critical Reviews in Biomedical Engineering (Editor-John R Bourne, Vanderbitt University, Nashville, TN 37232, USA 25(2): 120-125.

4. Festing Simo, Wilkinson Robin (2007) The ethics of animal research: ЕМBO Rep 8(6): 526-530.

5. Pal TK (2015) Animal Experimentation: Part I- General Considerations. J Int Clin Dent Res Organ 7: 7-10.

6. Pal TK (2015) Animal experimentation-Part-II: In periodontal research. J Int Clin Dent Res Organ 1(7): 92-99.

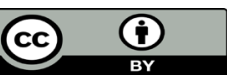

This work is licensed under Creative Commons Attribution 4.0 License

To Submit Your Article Click Here: Submit Article

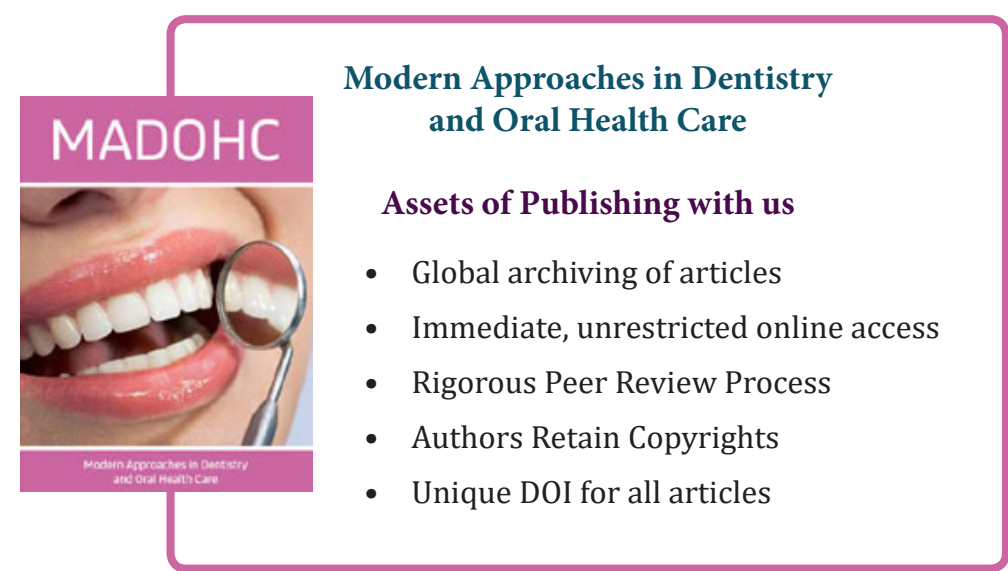

